\title{
Safety and efficacy of venoplasty in MS
}

\section{A randomized, double-blind, sham-controlled phase II trial}

Anthony L. Traboulsee, MD, Lindsay Machan, MD, J. Marc Girard, MD, Jean Raymond, MD, Reza Vosoughi, MD, Brian W. Hardy, MD, Francois Emond, MD, Jean-Luc Gariepy, MD, Jeffrey N. Bone, MSc, Gary Siskin, MD, Darren Klass, MD, Saul Isserow, MD, Judy Illes, PhD, A. Dessa Sadovnick, PhD, and David K. Li, MD

Neurology ${ }^{\circledR}$ 2018;91:e1660-e1668. doi:10.1212/WNL.0000000000006423

\section{Abstract}

\section{Objective}

To determine the safety and efficacy of balloon vs sham venoplasty of narrowing of the extracranial jugular and azygos veins in multiple sclerosis (MS).

\section{Methods}

Patients with relapsing or progressive MS were screened using clinical and ultrasound criteria. After confirmation of $>50 \%$ narrowing by venography, participants were randomized 1:1 to receive balloon or sham venoplasty of all stenoses and were followed for 48 weeks. Participants and research staff were blinded to intervention allocation. The primary safety outcome was the number of adverse events (AEs) during 48 weeks. The primary efficacy outcome was the change from baseline to week 48 in the patient-reported outcome MS Quality of Life-54 (MSQOL-54) questionnaire. Standardized clinical and MRI outcomes were also evaluated.

\section{Results}

One hundred four participants were randomized ( 55 sham; 49 venoplasty) and 103 completed 48 weeks of follow-up. Twenty-three sham and 21 venoplasty participants reported at least 1 $\mathrm{AE}$; one sham $(2 \%)$ and $5(10 \%)$ venoplasty participants had a serious AE. The mean improvement in MSQOL-54 physical score was +1.3 (sham) and +1.4 (venoplasty) $(p=0.95)$; MSQOL-54 mental score was +1.2 ( sham) and -0.8 (venoplasty) $(p=0.55)$.

\section{Conclusions}

Our data do not support the continued use of venoplasty of extracranial jugular and/or azygous venous narrowing to improve patient-reported outcomes, chronic MS symptoms, or the disease course of MS.

\section{ClinicalTrials.gov identifier NCT01864941.}

\section{Classification of evidence}

This study provides Class I evidence that for patients with MS, balloon venoplasty of extracranial jugular and azygous veins is not beneficial in improving patient-reported, standardized clinical, or MRI outcomes.

\author{
Correspondence \\ Dr. Traboulsee \\ t.traboulsee@ubc.ca
}

\section{RELATED ARTICLE}

\section{Editorial}

Venoplasty in MS:

Therapeutic intervention without any evidence

Page 815

\section{MORE ONLINE}

$\rightarrow$ Class of Evidence
Criteria for rating
therapeutic and diagnostic
studies
NPub.org/coe

$\rightarrow$ Class of Evidence

Criteria for rating studies

NPub.org/coe 


\section{Glossary}

AE = adverse event; CCSVI = chronic cerebrospinal venous insufficiency; CUA = combined unique active; DMT = diseasemodifying therapy; EDSS = Expanded Disability Status Scale; IJV = internal jugular vein; $\mathbf{M S}=$ multiple sclerosis; $\mathbf{M S F C}=$ Multiple Sclerosis Functional Composite; MSQOL-54 = Multiple Sclerosis Quality of Life-54; PRO = patient-reported outcome; RRMS = relapsing-remitting multiple sclerosis; $\mathrm{SAE}=$ serious adverse event; UBC $=$ University of British Columbia.

Multiple sclerosis (MS) is an inflammatory and degenerative demyelinating disease of the human CNS. While abundant scientific and clinical data support the involvement of the immune system in the pathogenesis of MS, ${ }^{1}$ the etiology and management of many clinical manifestations of the disease (e.g., fatigue and cognitive impairment) remain poorly understood. Chronic cerebrospinal venous insufficiency (CCSVI), described as a combination of extracranial venous structural and flow anomalies, had been proposed as contributing to the pathogenesis and disabling symptoms ${ }^{2}$ in MS, and subsequently in other conditions including Alzheimer disease, ${ }^{3}$ Parkinson disease, ${ }^{4}$ and Ménière disease. ${ }^{5}$ It had been postulated that impaired venous drainage would cause stasis leading to perivenular iron deposition triggering inflammation. This pathogenic hypothesis remains poorly supported, and venous narrowing has been found as frequently in healthy volunteers as in patients with MS. ${ }^{6}$ Multiple uncontrolled, unblinded studies and case series suggested that venous dilation with or without intravascular stenting could improve symptoms and modify the disease course in $\mathrm{MS}^{2,7-15}$ and Ménière disease. ${ }^{5}$ These procedures were promoted through social media and were readily available through medical tourism ${ }^{16}$ despite warnings by regulatory bodies including the US Food and Drug Administration about significant complications. ${ }^{17}$

We conducted a randomized, sham-controlled, double-blind, interventional trial in relapsing-remitting and progressive forms of $\mathrm{MS}^{18}$ to determine short- and long-term safety and efficacy on patient-reported outcomes (PROs), and standard clinical and MRI outcomes up to 48 weeks.

\section{Methods}

\section{Participants}

Between May 29, 2013, and August 19, 2015, we recruited participants with relapsing-remitting MS (RRMS), secondary progressive MS, and primary progressive MS at 4 Canadian academic centers with MS clinics and interventional radiology expertise (University of British Columbia [UBC] Hospital, Vancouver; Health Sciences Centre, Winnipeg; CHUM, Hôpital Notre-Dame, Montreal; Hôpital Enfant-Jesus, Québec). The following were inclusion criteria: age 18 to 65 years, diagnosis of definite MS by the $2010 \mathrm{McD}$ onald criteria, ${ }^{19}$ an Expanded Disability Status Scale (EDSS) ${ }^{20}$ score between 0 (i.e., minimal disability) and 6.5 (i.e., using bilateral aids to walk), neurologically stable disease within the 30 days before screening. In addition, participants fulfilled at least 2 ultrasound criteria for CCSVI defined by the Canadian Institutes of Health Research Imaging Expert Panel (appendix e-1, links.lww.com/WNL/A720): reflux in the internal jugular vein (IJV) and/or vertebral vein; reflux present in the deep cerebral veins; B mode IJV stenosis; absent IJV and/or vertebral vein flow; and negative IJV cross-sectional area. Participants had to have confirmation on catheter venography of $>50 \%$ narrowing affecting at least 1 of 3 extracranial veins (either internal jugular and/or azygos). ${ }^{6}$ Participants on standard disease-modifying therapies (DMTs) were permitted to continue on medication, and changes were allowed for on-study relapses after randomization.

Exclusion criteria included treatment with vasodilators, parasympathomimetics, sympathicolytics, calcium channel blockers, previous venoplasty and/or stenting, previous jugular or subclavian central line or major neck surgery or radiation, previous contrast allergy, inability to undergo MRI, inadequate medical records confirming diagnosis and disease course, and inability to complete all study visits.

After randomization and intervention, participants were followed for 48 weeks with adverse event (AE) assessments, standardized PRO scales (72 hours and weeks 2, 12, 24, 36, and 48), Multiple Sclerosis Functional Composite (MSFC) ${ }^{21}$ and EDSS scores (weeks 2, 12, 24, and 48), and MRI and ultrasound (weeks 24 and 48 ) assessments.

\section{Standard protocol approvals, registrations, and patient consents}

The clinical research ethics boards at the 4 participating centers approved the study protocol. The trial was registered with clinicaltrials.gov NCT01864941.

\section{Procedures}

Personnel at all sites underwent standardized training for ultrasound (L.M.), venography (L.M.), and venoplasty (L.M. and G.S.) procedures. Venography was performed under conscious sedation, and the duration of time within the angiography suite was uniform for both venoplasty- and sham-treated participants. A 5-French diagnostic catheter was introduced through the common femoral vein to selectively catheterize the right and left IJVs as well as the azygos vein using a standardized protocol. ${ }^{6}$ Venous stenosis was determined by dividing the minimum (narrowest) lumen diameter by the reference lumen diameter (normal vein diameter proximal or distal to the stenosis). ${ }^{6}$ Participants with $>50 \%$ narrowing of any of the 3 veins were randomized $(1: 1)$ to either sham or active balloon venoplasty of all 
narrowed veins under study. The venoplasty participants were treated with an angioplasty balloon $2 \mathrm{~mm}$ greater than the nominal vein diameter, which was inflated for $60 \mathrm{sec}-$ onds. A repeat venoplasty was performed for persistent narrowing $>50 \%$. The participants randomized to sham had a catheter that was advanced across the stenosis and left for 60 seconds.

\section{Randomization and masking}

Stratified treatment randomization (RRMS vs progressive MS course $^{18}$ ) at each site was completed by a permuted-block size of 6 to reduce the likelihood of obtaining unbalanced groups. The randomization table was generated by an independent statistician. Up to a maximum of $50 \%$ progressive disease was required for enrollment per site. Treatment assignments were sealed in individual envelopes, only opened after eligibility was confirmed, and resealed after the procedure. All participants and assessors were blinded to intervention assignment. The interventional team was not involved in any outcome assessments.

\section{Outcomes}

The primary safety outcome included all AEs that are clinically significant and/or potentially related to study procedure and/or serious up to week 48 , as determined by the principal investigators and in accordance with the International Conference on Harmonization. ${ }^{22}$ The primary efficacy outcome was the change in the MS Quality of Life-54 (MSQOL-54) physical and mental composite scores $^{23}$ from baseline to week 48 . Secondary efficacy outcomes were the changes in MSQOL-54 physical and mental composite from baseline to 72 hours to ensure an early, transient effect was not missed; Fatigue Severity Scale, ${ }^{24}$ North American Research Committee on MS pain scale, ${ }^{25}$ and CCSVI symptom scale ${ }^{26}$ from baseline to 72 hours and week 48; and change in EDSS (median) and MSFC (mean) from baseline to week 48. Protocol-defined relapses were a 1-point increase in EDSS or a 1-point change on any functional status score representative of the relapse location. Combined unique active (CUA) lesions, defined as a contrast enhancing lesion on $\mathrm{T} 1$-weighted scan or a non-T1 enhancing, new/enlarging T2 lesion, compared to the previous MRI were assessed by the UBC MS/MRI Research Group from brain MRIs with contrast performed at baseline, week 24 , and week 48 .

\section{Oversight}

Study data were collected and managed using REDCap hosted at the British Columbia Children's Hospital Research Institute. ${ }^{27}$ Independent clinical research associates monitored source documents, case report forms, and database entries prior to database lock. An independent medical monitor (S.I.: cardiologist, UBC) reviewed all serious AEs (SAEs). An independent data safety monitoring board, which included a vascular surgeon, neurologist, ethicist, and statistician, reviewed AEs and trial progress.

\section{Statistical analyses}

The number of participants to be included in this phase II trial was based on the primary efficacy outcome, a mean change in MSQOL-54 composite scores from baseline to week 48. Assuming an SD of $20,{ }^{23} 40$ participants per group would give $60 \%$ power to demonstrate a difference of 10 points on the MSQOL-54 composite score (an effect size of 10/20 =0.5) using a 2-tailed $t$ test at the significance level of 0.05 . At least 100 participants were randomized anticipating a potential withdrawal rate as high as $20 \%$. The analysis plan was developed before database lock and unblinding. Analyses (safety and efficacy) were performed on the intention-to-treat population (all patients randomized) and based on data collected up to week 48. No imputation of missing values was performed. If individual patients had missing values for a particular outcome, those patients were not included in the analysis of that outcome. For the primary safety outcome (significant AEs, SAEs, and venography complications up to week 48), the distribution of the total number of AEs per participant between groups was compared with the Fisher exact test (noncontinuous variable). We also applied negative binomial regression analysis to the number of AEs per participant to estimate the relative rate; logistic regression to compare the proportion of participants having $\geq 1 \mathrm{AE}$; and more detailed versions of the regression analyses adjusting for covariates.

The changes from baseline for the efficacy outcomes (PROs, MSFC, and EDSS) were treated as continuous outcomes compared using a Student 2-sample $t$ test. Additional regression analyses used the baseline level of the outcome as a covariate. At each follow-up time, PROs and MSFC were dichotomized as "improved" or "not improved" relative to the participant's baseline value and compared between groups using the Fisher exact test and logistic regression. Protocoldefined relapses were compared using the Fisher exact test and logistic regression analysis, and MRI CUA lesions were analyzed using the Fisher test and negative binomial regression.

The trial was conducted in accordance with the provisions of the International Conference on Harmonization Guidelines for Good Clinical Practice and the Declaration of Helsinki. ${ }^{22}$ This study provides Class I evidence for assessing whether balloon venoplasty of extracranial jugular and azygous veins is beneficial in improving PROs, standardized clinical, and MRI outcomes in patients with relapsing or progressive MS.

\section{Data availability}

Anonymized data can be made available to qualified investigators upon request to the corresponding author.

\section{Results}

\section{Screening}

The first participant was randomized on May 29, 2013, and the last participant completed week 48 on July 28,2016 . We 
screened 274 patients, of whom 104 were randomized (49 venoplasty, 55 sham) across the 4 centers (figure 1). Failure to meet ultrasound criteria $(\mathrm{n}=82)$, medical contraindications $(\mathrm{n}=33)$, MRI or venography contraindications $(\mathrm{n}=3)$, or insufficient medical records $(n=4)$ accounted for the triage of 122 patients who volunteered to participate. Sixteen participants who were potentially eligible for enrollment declined to participate in further screening with venography. One hundred thirty-six participants had venography performed, and of these, 104 (76\%) fulfilled the final enrollment criteria $(>50 \%$ narrowing) and were randomized. Overall retention to week 48 was 103 participants (98\%). One participant randomized to sham treatment withdrew at week 24 because of a time conflict. One participant randomized to sham only completed MRI and blood work at week 48 .

\section{Baseline}

The mean age at enrollment was 50.5 years (range 33-65) with a mean disease duration of 17 years; $65 \%$ of participants were women (68/104) and 62\% (64/104) had RRMS (table 1). Sixty-nine percent of participants with RRMS (44/64) were on DMTs. Characteristics were similar between treatment groups and centers. On baseline venography, 50\% of participants (32/64) with RRMS and 72\% (29/40) with secondary progressive or primary progressive MS had multiple vessels with $>50 \%$ narrowing.

\section{Efficacy: PROs}

There was a transient increase in MSQOL scores within 72 hours (mental scores) and 2 weeks (physical scores) in both groups. The mean improvement from baseline to week 48 for MSQOL physical score was +1.3 and +1.4 (sham vs venoplasty $p=0.95$ ); MSQOL mental score +1.2 and -0.8 (sham vs venoplasty $p=0.55$ ); fatigue score +0.2 and +0.1 (sham vs venoplasty $p=0.65$ ); and pain score was +0.1 and -0.2 (sham vs venoplasty $p=0.19$ ). There was no significant difference in the proportion of sham and venoplasty participants who had an improvement in a PRO from baseline to week 48 (figure 2). There was no difference between sham and venoplasty groups on all PROs at 72 hours post procedure (table 2; table e-1, links.lww.com/WNL/A719). Improvements in the CCSVI symptom scale for limb temperature occurred in $6 / 50(12 \%)$ sham and $4 / 48(8 \%)$ venoplasty $(p=0.74)$, limb color in $5 / 50(10 \%)$ sham and

Figure 1 Trial profile

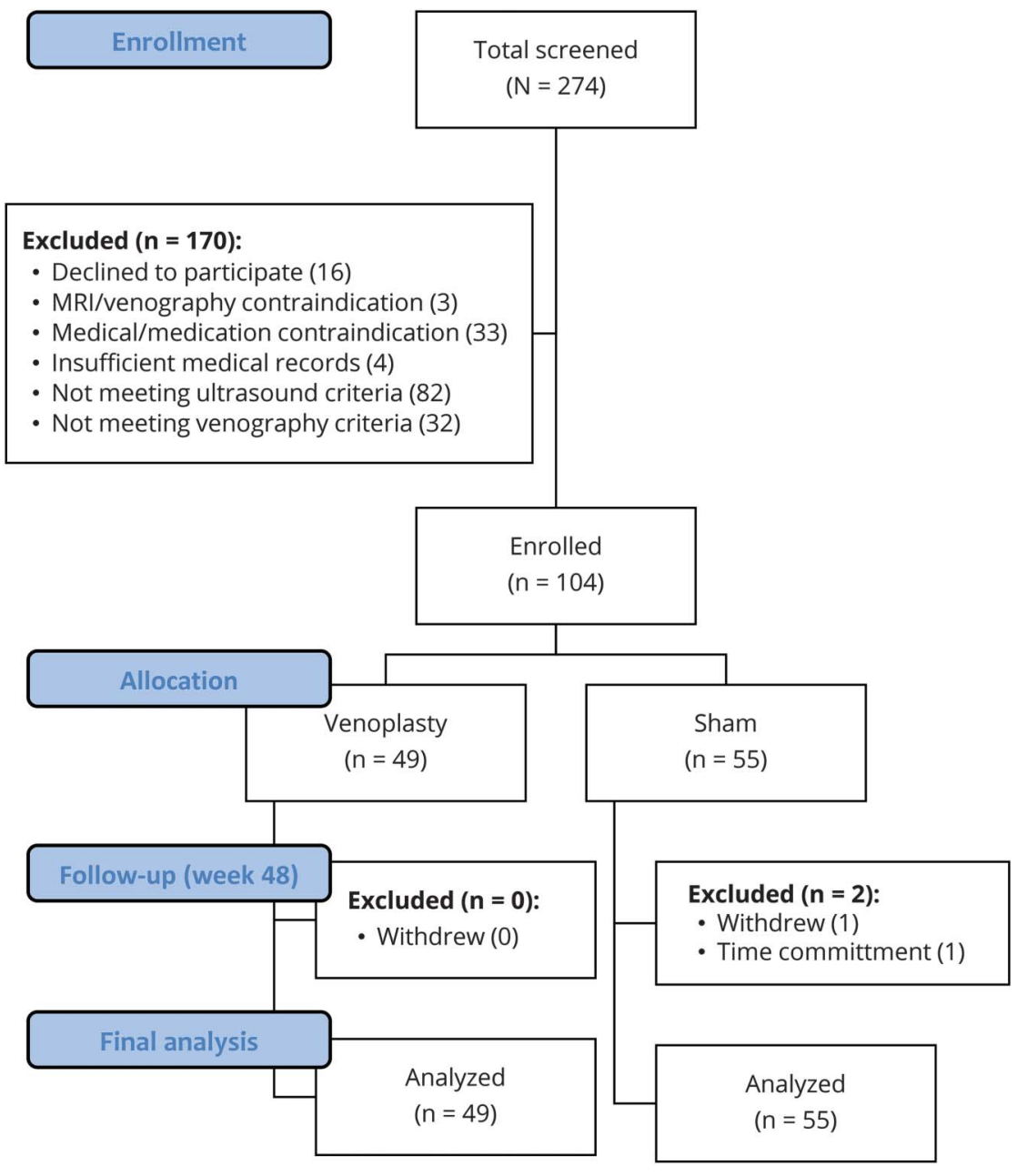


Table 1 Baseline demographics

\begin{tabular}{|c|c|c|c|c|}
\hline Characteristic & Sham $(n=55)$ & Venoplasty $(n=49)$ & All $(n=104)$ & $p$ Value $^{a}$ \\
\hline Age, $y$, mean (range) & $50.1(33-65)$ & $51.0(35-63)$ & $50.5(33-65)$ & 0.54 \\
\hline Female, n (\%) & $34(62)$ & $34(69)$ & $68(65)$ & 0.54 \\
\hline Disease course, $\mathbf{n}(\%)$ & & & & 1.00 \\
\hline Relapsing-remitting & $34(62)$ & $30(61)$ & $64(62)$ & \\
\hline Progressive & $21(38)$ & $19(39)$ & $40(38)$ & \\
\hline Disease duration, $y$, mean (range) & $16.2(4-40)$ & $18.1(3-41)$ & $17.1(3-41)$ & 0.30 \\
\hline EDSS score, median (range) & $4(0-6.5)$ & $4(0-6.5)$ & $4(0-6.5)$ & 0.65 \\
\hline Disease-modify therapy, $\mathrm{n}(\%)$ & $24(43.6)$ & $20(40.8)$ & $44(42.3)$ & 0.84 \\
\hline Interferon beta-1a or $-1 \mathrm{~b}$ & $10(18.2)$ & $6(12.2)$ & $16(15.4)$ & \\
\hline Glatiramer acetate & $7(12.7)$ & $7(14.3)$ & $14(13.5)$ & \\
\hline Oral $^{b}$ & $7(12.7)$ & $5(10.2)$ & $12(11.5)$ & \\
\hline Natalizumab & 0 & $2(4.1)$ & $2(1.9)$ & \\
\hline Relapses in past year, n (\%) & & & & 1.00 \\
\hline 0 & $48(87.3)$ & $43(87.8)$ & $91(87.5)$ & \\
\hline 1 & $6(10.9)$ & $6(12.2)$ & $12(11.5)$ & \\
\hline 2 & $1(1.8)$ & 0 & $1(1.0)$ & \\
\hline MRI with T1 enhancing lesions, $n(\%)$ & $9(16.4)$ & $4(8.5)^{c}$ & $13(12.7)$ & 0.37 \\
\hline Venography, $n$ (\%) & & & & $0.16^{d}$ \\
\hline Single vessel $>50 \%$ narrowing & $19(34.5)$ & $24(49.0)$ & $43(41.3)$ & \\
\hline Either jugular & $15(27.3)$ & $23(46.9)$ & $38(36.5)$ & \\
\hline Azygos only & $4(7.3)$ & $1(2.0)$ & $5(4.8)$ & \\
\hline Multiple vessels $>\mathbf{5 0} \%$ narrowing & $36(65.5)$ & $25(51.0)$ & $61(58.7)$ & \\
\hline Both jugulars & $22(40.0)$ & $21(42.9)$ & $43(41.3)$ & \\
\hline One jugular and azygos & $6(11.0)$ & $3(6.1)$ & $9(8.7)$ & \\
\hline All 3 vessels & $8(14.5)$ & $1(2.0)$ & $9(8.7)$ & \\
\hline \multicolumn{5}{|c|}{$\begin{array}{l}\text { Abbreviation: EDSS = Expanded Disability Status Scale. } \\
\text { a Student 2-sample } t \text { test for continuous variables, Fisher exact test for categorical variables. } \\
\text { b Oral disease-modifying therapies include teriflunomide, dimethyl fumarate, and fingolimod. } \\
\text { " Two patients were missing these data. } \\
\text { " Reflects test of "single" vessel vs "multiple" vessels (not further subclassifications). }\end{array}$} \\
\hline
\end{tabular}

$3 / 48(6 \%)(p=0.71)$ venoplasty, brain fog in $9 / 50(18 \%)$ sham and $7 / 48(15 \%)$ venoplasty $(p=0.59)$, and visual function in 6/50 (12\%) sham and 3/48 (6\%) venoplasty $(p=0.49)$.

\section{Efficacy: Clinical and MRI outcomes}

There was no improvement in MSFC from baseline to week 48 for the sham $(0.0)$ or venoplasty $(-0.4)$ group $(p=0.18)$. There was little change in median EDSS score at week 48 in either group. Eleven participants ( 6 sham, 5 venoplasty) had a protocol-defined relapse $(p=0.91)$; and 21 and 18 participants, respectively, had at least one MRI CUA lesion $(p=0.88)$ (table 3$)$. Of the $44(24 / 55$ sham, $20 / 50$ venoplasty) participants on DMT at baseline, 1 sham participant discontinued DMT, 3 (1 sham, 2 venoplasty) switched DMTs, and 1 venoplasty participant had a temporary interruption of DMT use. Two sham participants were started on a DMT after baseline. The mean percent change from baseline to week 48 in brain volume was -0.693 and -0.707 $(p=0.93)$ for sham and venoplasty groups.

Subgroup analysis of participants with the presence or absence of gadolinium-enhancing lesions at baseline, relapsing or progressive disease course, or the presence or absence of 
Table 2 Improvements in patient-reported outcomes from baseline to 72 hours

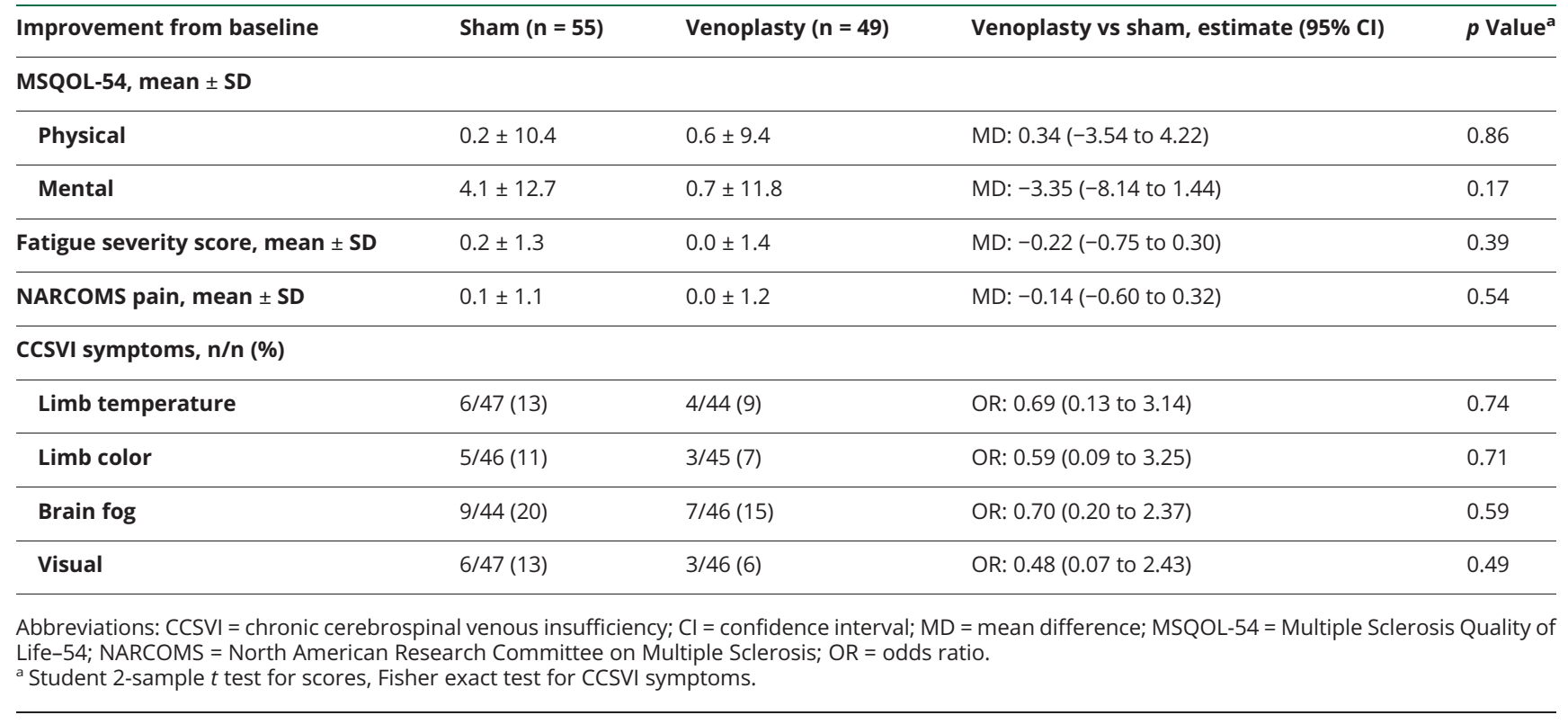

azygos stenosis could not detect a benefit of venoplasty compared to sham on any outcome.

\section{Safety}

No deaths occurred during the study. No periprocedural (within 48 hours) SAEs requiring hospitalization were reported. There was one asymptomatic internal jugular dissection that occurred in the venoplasty group that did not require intervention or hospitalization. Three (5\%) sham and $3(6 \%)$ venoplasty participants reported moderate or severe pain during the procedure $(p=0.88) ; 6 / 54(11 \%)$ and $4 / 49$ $(8 \%)$, respectively, reported postprocedure pain $(p=0.62)$. Twenty of the 55 (36\%) sham participants reported 37 AEs and $17 / 49$ (35\%) venoplasty participants reported 22 AEs within 48 hours. The most commonly reported periprocedural AEs were groin pain $(\mathrm{n}=8 / 104,7.7 \%)$, hematoma $(\mathrm{n}=$ $9 / 104,8.6 \%)$, and neck pain $(\mathrm{n}=6 / 106,5.7 \%)$.

\section{AE to week 48}

The number of participants with any AEs reported from baseline to week 48 was $42 \%(23 / 55)$ for sham and $43 \%$ (21/49) for venoplasty ( $p=1$, Fisher exact test) (table e-2, links.lww.com/WNL/A719). The most commonly reported AEs were gastrointestinal reflux or discomfort $(n=8)$, paresthesia and/or lightheadedness $(n=8)$, arthralgia $(n=6)$, and general malaise $(n=4)$. There were no cases of venous thrombosis up to week 48. Six (5.7\%) SAEs were reported $(1 / 55$ [2\%] sham, $5 / 49$ [10\%] venoplasty), none of which were related to the study procedure in the opinion of the blinded physician. The SAEs were generalized seizure ( 1 sham, week 17), sepsis (2 venoplasty, weeks 20 and 25), bleeding of a previously undiagnosed cerebral aneurysm (1 venoplasty, week 46), myocardial infarction (1 venoplasty, week 28 ), and pulmonary embolism (1 venoplasty, week 17$)$.

\section{Discussion}

Before commencing this study in 2013, there were no randomized, blinded, sham-controlled trials on this topic reported since the first open-label study of 65 patients in 2009 . $^{2}$ Eight additional open-label, uncontrolled, prospective or retrospective case series involving 1,655 patients (range $15-1,202)$ and unblinded evaluators have been reported. ${ }^{7-15}$ The majority of these reported improvement in MS symptoms with PRO scales including MSQOL-54 and/or the EDSS. Subsequently, a retrospective cohort of 462 patients at 33 Italian centers ${ }^{28}$ and a small prospective randomized study of 19 participants with MS (10 sham, 9 venoplasty) did not detect any benefit of venoplasty on clinical and MRI outcomes. ${ }^{29}$

We chose not to use vascular stents because of concerning reports of serious complications including stent thrombosis, ${ }^{30}$ stent embolization, and death. ${ }^{15}$ However, venoplasty alone can also be associated with SAEs, necessitating careful monitoring in this trial. ${ }^{31}$ The original open-label venoplasty trial did not use intravascular stents and reported an improvement in symptoms during 18 months of follow-up. ${ }^{2}$ We hypothesized that venoplasty without stenting should be sufficient to detect efficacy within 48 weeks without putting patients at greater risk of complications related to intravascular stents. Our findings support this decision, given that significant AEs were similar between the sham and venoplasty groups at 48 hours and 48 weeks post procedure, with no evidence of thrombosis up to week 48 . However, SAEs were numerically higher in the venoplasty group at $10 \%$ vs $2 \%$ for sham.

There are important MS symptoms, including fatigue and pain, that are not adequately captured by routinely used clinician-assessed scales (EDSS and MSFC). Our study 

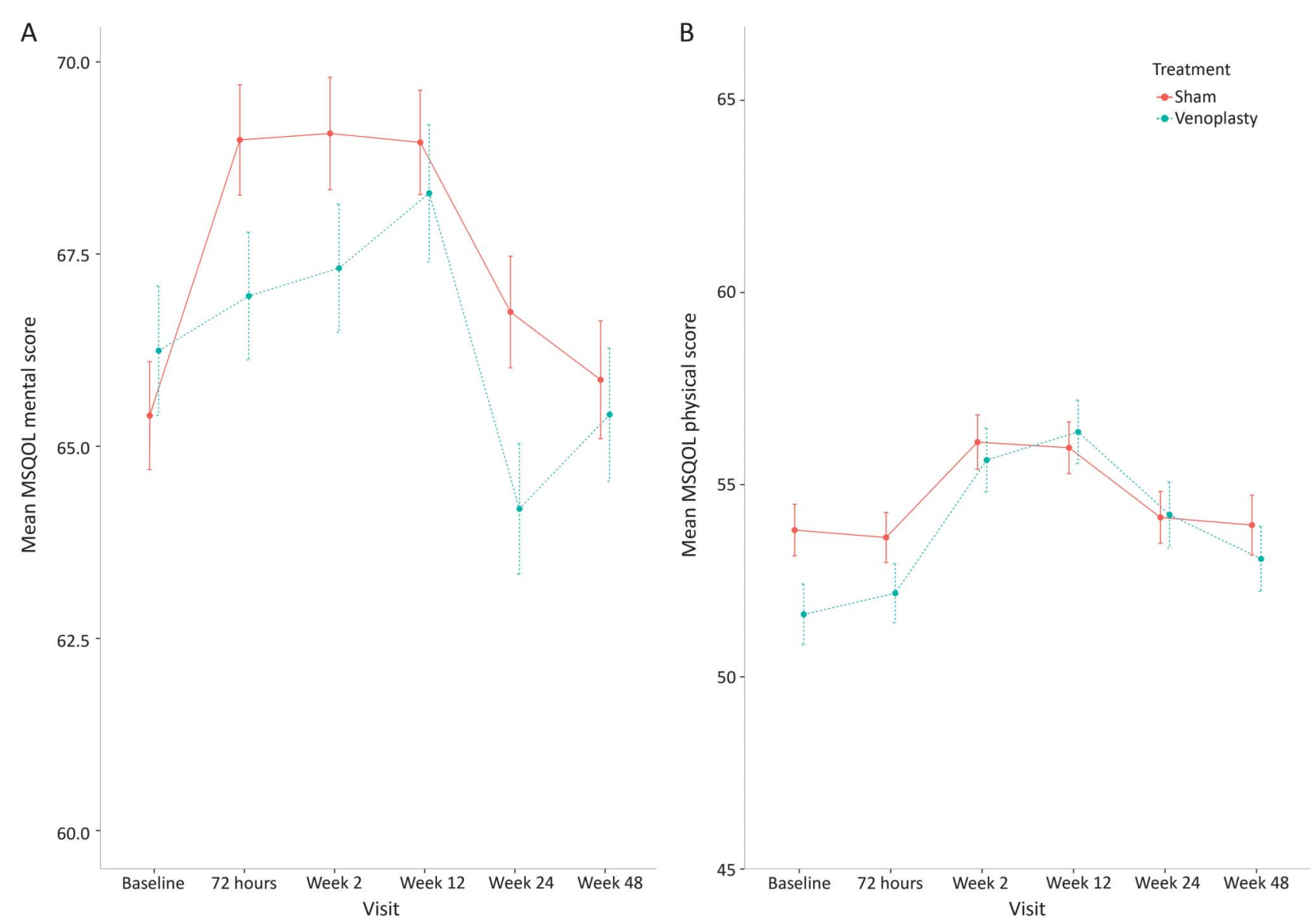

A higher scale score indicates a higher quality of life. A similar, transient increase in scores was seen in the venoplasty and sham groups. MSQOL-54= Multiple Sclerosis Quality of Life-54.

design ensured that we could determine whether the intervention had an early improvement on outcomes meaningful to patients as measured using validated PROs and a novel CCSVI symptom scale. Participants were reassessed over 48 weeks to determine whether any early improvements in symptoms were sustained, and to detect any delayed improvements that may have been masked by periprocedural pain or residual effects of the medications used for conscious sedation. There were no differences between the sham and venoplasty groups on the PROs throughout the 48 weeks. While it is possible that our study was underpowered to detect statistically significant differences, we had surpassed our target enrollment, 99\% retention, and our cohort size was larger than the original open-label study. ${ }^{2}$ We could not detect any trends in any of the PROs and either clinical or MRI outcomes.

There was no difference compared to the sham intervention or in the number of participants who had objective evidence of new inflammatory activity (MRI CUA lesion and/or confirmed clinical relapses) or improvement in disability (EDSS or MSFC). This would argue against venoplasty having a disease-modifying mechanism of action.
We took great care in standardizing the venography and intervention procedures across the 4 centers. Stratified randomization by disease type at each site reduced the likelihood of obtaining unbalanced study groups. The conscious sedation during venography, and a standardized venography procedure room experience for all participants (i.e., room setup, duration of time in the room, minimal staff present), ensured that all sham and venoplasty participants would have an identical research experience to optimize masking.

Venous narrowing $>50 \%$ was present in $76 \%$ of participants, similar to earlier findings in $\mathrm{MS}^{6}{ }^{6} \mathrm{We}$ included participants with the progressive form of MS, i.e., those with the greatest unmet medical need for treatments that could significantly improve quality of life. Early reports ${ }^{2}$ suggested that patients with RRMS were more likely to benefit from venoplasty than patients with progressive MS, but our subgroup analyses did not detect outcome differences between sham and venoplasty for either RRMS or progressive MS patients.

Extracranial venous stenosis continues to be proposed as a pathogenic disorder for a variety of neurologic conditions 
Table 3 Improvements in patient-reported and clinical outcomes from baseline to week 48; disease progression, confirmed relapses, and new MRI disease activity from baseline to week 48

\begin{tabular}{|c|c|c|c|c|}
\hline Outcome & Sham $(n=55)$ & Venoplasty $(n=49)$ & Venoplasty vs sham, estimate $(95 \% \mathrm{Cl})$ & $p$ Value $^{a}$ \\
\hline \multicolumn{5}{|l|}{ MSQOL-54, mean \pm SD } \\
\hline Physical & $1.3 \pm 11.5$ & $1.4 \pm 14.0$ & MD: 0.17 ( -4.88 to 5.22$)$ & 0.95 \\
\hline Mental & $1.2 \pm 15.2$ & $-0.8 \pm 18.7$ & MD: $-2.02(-8.75$ to 4.71$)$ & 0.55 \\
\hline Fatigue severity score, mean \pm SD & $0.2 \pm 1.65$ & $0.1 \pm 1.2$ & MD: $-0.13(-0.70$ to 0.44$)$ & 0.65 \\
\hline NARCOMS pain, mean \pm SD & $0.1 \pm 1.7$ & $-0.2 \pm 1.0$ & MD: $-0.30(-0.74$ to 0.15$)$ & 0.19 \\
\hline MSFC, mean \pm SD & $0.0 \pm 0.7$ & $-0.4 \pm 1.5$ & MD: $-0.39(-0.88$ to 0.10$)$ & 0.12 \\
\hline EDSS, median \pm SD & $0.0 \pm 0.9$ & $-0.2 \pm 0.8$ & MD: 0.15 (-0.18 to 0.49$)$ & 0.36 \\
\hline 3-Mo confirmed disability progression, $n$ (\%) & $3(5)$ & $1(2)$ & OR: 0.36 (0.01 to 4.71$)$ & 0.62 \\
\hline Participants with relapses, $\mathrm{n}(\%)$ & $6(11)$ & $5(10)$ & OR: 0.93 (0.21 to 3.94$)$ & 1.00 \\
\hline Participants with MRI CUA lesions, n (\%) & & & RR: 0.27 (0.09 to 0.83$)$ & 0.32 \\
\hline 1 CUA & $8(13)$ & $3(5)$ & & \\
\hline 2 CUA & $4(9)$ & $2(4)$ & & \\
\hline 3 CUA & $1(7)$ & $0(0)$ & & \\
\hline 4 CUA & $2(4)$ & $4(8)$ & & \\
\hline $5+$ CUA & $4(6)$ & $2(4)$ & & \\
\hline Not available & $3(5)$ & $2(4)$ & & \\
\hline
\end{tabular}

Abbreviations: $\mathrm{Cl}$ = confidence interval; $\mathrm{CUA}=$ combined unique active; $\mathrm{EDSS}=$ Expanded Disability Status Scale; $\mathrm{MD}=$ mean difference; $\mathrm{MSFC}=\mathrm{Multiple}$ Sclerosis Functional Composite; MSQOL-54 = Multiple Sclerosis Quality of Life-54; NARCOMS = North American Research Committee on Multiple Sclerosis; OR = odds ratio; $\mathrm{RR}=$ relative rate.

a Student 2-sample $t$ test for scores, Fisher exact test for categorical outcomes.

(including Alzheimer, Parkinson, and Ménière diseases). It is not uncommon for patients to experience improvements in their symptoms with intervention, especially when expectations are high. Sham-controlled confirmatory trials are rarely performed for interventional procedures, but they remain necessary. Uncontrolled case series can erroneously suggest a benefit from novel but ineffective therapies. ${ }^{32}$ This large randomized, double-blind, multicenter clinical trial failed to show superiority of venoplasty compared to sham intervention on patient-reported, clinical, and MRI outcomes.

In the face of extraordinary pressure to find a cure for neurodegenerative diseases, this study confirms the importance of well-designed trials to assess the safety and effectiveness of new therapies, including those that may be publicly popular and perhaps less conventional. It is critical to ensure that patients do not undergo procedures that could result in unnecessary costs or morbidity, or lose trust and hope in the vital goals of biomedical research.

\section{Author contributions}

A.L. Traboulsee: literature search, tables, study design, data collection, data interpretation, writing, and final approval of the manuscript. L. Machan, D.K. Li, A.D. Sadovnick, S. Isserow, J. Illes, D. Klass, J.M. Girard, J. Raymond, R. Vosoughi,
B.W. Hardy, G. Siskin, F. Emond, and J.-L. Gariepy: study design, data collection, data interpretation, and final approval of the manuscript. J.N. Bone: statistical analysis, data interpretation, writing, and final approval of the manuscript.

\section{Acknowledgment}

The authors are grateful to the participants in the trial; patient advocates for reviewing and contributing to study design; the independent data safety monitoring board members Dr. Michael Yeung, Dr. David Taylor, Prof. John Petkau, and the late Prof. David Roy; the clinical trial staff; the interventional and postprocedure care teams; and the late Mr. Rick White (statistical support) for their invaluable contributions.

\section{Study funding}

The study was primarily supported by cooperative agreements from the Canadian Institutes of Health Research (CIHR), MS Society of Canada, Michael Smith Foundation for Health Research, Research Manitoba, and Ministère de la Santé et des Services Sociaux du Québec. The study design was peer reviewed and approved by CIHR.

\section{Disclosure}

A.L. Traboulsee reports grants and/or personal fees from Biogen Idec, Chugai, Canadian Institutes of Health Research, 
Hoffmann-La Roche, Multiple Sclerosis Society of Canada, Sanofi Genzyme, Novartis, Teva Innovation, Consortium of MS Centers. L. Machan reports grants from Canadian Institutes of Health Research, Michael Smith Foundation for Health Research, Multiple Sclerosis Society of Canada during the conduct of the study. J.M. Girard reports grants from Canadian Institutes of Health Research, Ministère de la Santé et des Services Sociaux du Québec, Multiple Sclerosis Society of Canada during the conduct of the study; grants and personal fees from Biogen, Hoffmann-La Roche, Genzyme, personal fees from Novartis, EMD Serono, grants from $A B$ Science outside the submitted work. J. Raymond reports grants from Canadian Institutes of Health Research, Ministère de la Santé et des Services Sociaux du Québec, Multiple Sclerosis Society of Canada during the conduct of the study. R. Vosoughi reports grants from Canadian Institutes of Health Research, Research Manitoba, Multiple Sclerosis Society of Canada during the conduct of the study. B.W. Hardy reports grants from Canadian Institutes of Health Research, Research Manitoba, Multiple Sclerosis Society of Canada during the conduct of the study. F. Emond reports grants from Canadian Institutes of Health Research, Ministère de la Santé et des Services Sociaux du Québec, Multiple Sclerosis Society of Canada during the conduct of the study; personal fees from Biogen Idec, EMD Serono, Teva Innovation Canada, grants and personal fees from Genzyme/Sanofi-Aventis, Novartis, Hoffmann-La Roche, outside the submitted work. J.-L. Gariepy reports no disclosures relevant to the manuscript. J.N. Bone reports grants from Canadian Institutes of Health Research, Michael Smith Foundation for Health Research, Multiple Sclerosis Society of Canada during the conduct of the study. G. Siskin reports no disclosures relevant to the manuscript. D. Klass reports grants from Canadian Institutes of Health Research, Michael Smith Foundation for Health Research, Multiple Sclerosis Society of Canada during the conduct of the study. S. Isserow reports grants from Canadian Institutes of Health Research, Michael Smith Foundation for Health Research, Multiple Sclerosis Society of Canada during the conduct of the study. J. Illes reports grants from Canadian Institutes of Health Research, Michael Smith Foundation for Health Research, Multiple Sclerosis Society of Canada during the conduct of the study. A.D. Sadovnick and D.K. Li report no disclosures relevant to the manuscript. Go to Neurology. org/ $\mathrm{N}$ for full disclosures.

\section{Publication history}

Received by Neurology September 29, 2017. Accepted in final form July 19, 2018.

\section{References}

1. Compston A, Coles A. Multiple sclerosis. Lancet 2008;372:1502-1517.

2. Zamboni P, Galeotti R, Menegatti E, et al. A prospective open-label study of endovascular treatment of chronic cerebrospinal venous insufficiency. J Vasc Surg 2009;50: 1348-1358.e3.

3. Beggs $\mathrm{C}$, Chung $\mathrm{CP}$, Bergsland $\mathrm{N}$, et al. Jugular venous reflux and brain parenchyma volumes in elderly patients with mild cognitive impairment and Alzheimer's disease. BMC Neurol 2013;13:157.
4. Liu M, Xu H, Wang Y, et al. Patterns of chronic venous insufficiency in the dural sinuses and extracranial draining veins and their relationship with white matter hyperintensities for patients with Parkinson's disease. J Vasc Surg 2015;61: 1511-1520.e1.

5. Bruno A, Napolitano M, Califano L, et al. The prevalence of chronic cerebrospinal venous insufficiency in Ménière disease: 24-month follow-up after angioplasty. J Vasc Interv Radiol 2017;28:388-391.

6. Traboulsee AL, Knox KB, Machan L, et al. Prevalence of extracranial venous narrowing on catheter venography in people with multiple sclerosis, their siblings, and unrelated healthy controls: a blinded, case-control study. Lancet 2014;383: $138-145$.

7. Zagaglia S, Balestrini S, Perticaroli E, et al. Percutaneous transluminal angioplasty for chronic cerebrospinal venous insufficiency in multiple sclerosis: dichotomy between subjective and objective outcome scores. Neurol Sci 2013;34:2205-2210.

8. Salvi F, Bartolomei I, Buccellato E, Galeotti R, Zamboni P. Venous angioplasty in multiple sclerosis: neurological outcome at two years in a cohort of relapsingremitting patients. Funct Neurol 2012;27:55-59.

9. Radak D, Kolar J, Sagic D, et al. Percutaneous angioplasty of internal jugular and azygous veins in patients with chronic cerebrospinal venous insufficiency and multiple sclerosis: early and mid-term results. Phlebology 2014;29:367-375.

10. Pryse-Phillips W, Stefanelli M, Murphy-Peddle K, Barrett B. An observational study of venoplasty in patients with multiple sclerosis. Can J Neurol Sci 2013;40:203-209.

11. Lupattelli T, Bellagamba G, Righi E, et al. Feasibility and safety of endovascular treatment for chronic cerebrospinal venous insufficiency in patients with multiple sclerosis. J Vasc Surg 2013;58:1609-1618.

12. Kostecki J, Zaniewski M, Ziaja K, et al. An endovascular treatment of chronic cerebrospinal venous insufficiency in multiple sclerosis patients: 6 month follow-up results. Neuro Endocrinol Lett 2011;32:557-562.

13. Hubbard D, Ponec D, Gooding J, Saxon R, Sauder H, Haacke M. Clinical improvement after extracranial venoplasty in multiple sclerosis. J Vasc Interv Radiol 2012;23: 1302-1308.

14. De Pasquale C, Pistorio ML, Veroux M, Giaquinta A, Veroux P, Fornaro M. Cognitive functioning and subjective quality of life in relapsing-remitting multiple sclerosis patients before and after percutaneous transluminal angioplasty: a preliminary report. Neuropsychiatr Dis Treat 2014;10:1039-1044.

15. Dake MD, Dantzker N, Bennett WL, Cooke JP. Endovascular correction of cerebrovenous anomalies in multiple sclerosis: a retrospective review of an uncontrolled case series. Vasc Med 2012;17:131-137.

16. Vera C, Herr A, Mandato K, Englander M, Ginsburg L, Siskin GP. Internet-based social networking and its role in the evolution of chronic cerebrospinal venous insufficiency. Tech Vasc Interv Radiol 2012;15:153-157.

17. Kuehn BM. FDA warns about the risks of unproven surgical therapy for multiple sclerosis. JAMA 2012;307:2575-2576.

18. Lublin FD, Reingold SC. Defining the clinical course of multiple sclerosis: results of an international survey. National Multiple Sclerosis Society (USA) Advisory Committee on Clinical Trials of New Agents in Multiple Sclerosis. Neurology 1996;46:907-911.

19. Polman CH, Reingold SC, Banwell B, et al. Diagnostic criteria for multiple sclerosis: 2010 revisions to the McDonald criteria. Ann Neurol 2011;69:292-302.

20. Kurtzke JF. Rating neurologic impairment in multiple sclerosis: an Expanded Disability Status Scale (EDSS). Neurology 1983;33:1444-1452.

21. Cutter GR, Baier ML, Rudick RA, et al. Development of a multiple sclerosis functional composite as a clinical trial outcome measure. Brain 1999;122(pt 5):871-882.

22. World Medical Association. World Medical Association Declaration of Helsinki: ethical principles for medical research involving human subjects. JAMA 2013;310: 2191-2194.

23. Vickrey BG, Hays RD, Harooni R, Myers LW, Ellison GW. A health-related quality of life measure for multiple sclerosis. Qual Life Res 1995;4:187-206.

24. Mills R, Young C, Nicholas R, Pallant J, Tennant A. Rasch analysis of the Fatigue Severity Scale in multiple sclerosis. Mult Scler 2009;15:81-87.

25. Marrie RA, Cutter G, Tyry T, Hadjimichael O, Vollmer T. Validation of the NARCOMS Registry: pain assessment. Mult Scler 2005;11:338-342.

26. Sadovnick AD, Yee IM, Attwell-Pope K, Keyes G, Kipp L, Traboulsee AL. Patientreported benefits of extracranial venous therapy: British Columbia CCSVI Registry. Can J Neurol Sci 2017;44:246-254.

27. Harris PA, Taylor R, Thielke R, Payne J, Gonzalez N, Conde JG. Research electronic data capture (REDCap): a metadata-driven methodology and workflow process for providing translational research informatics support. J Biomed Inform 2009;42: 377-381.

28. Ghezzi A, Annovazzi P, Cocco E, et al. Endovascular treatment of CCSVI in patients with multiple sclerosis: clinical outcome of 462 cases. Neurol Sci 2013;34:1633-1637.

29. Siddiqui AH, Zivadinov R, Benedict RH, et al. Prospective randomized trial of venous angioplasty in MS (PREMiSe). Neurology 2014;83:441-449.

30. Burton JM, Alikhani K, Goyal M, et al. Complications in MS patients after CCSVI procedures abroad (Calgary, AB). Can J Neurol Sci 2011;38:741-746.

31. Ghezzi A, Annovazzi P, Amato MP, et al. Adverse events after endovascular treatment of chronic cerebro-spinal venous insufficiency (CCSVI) in patients with multiple sclerosis. Mult Scler 2013;19:961-963.

32. Valdueza JM, Doepp F, Schreiber SJ, et al. What went wrong? The flawed concept of cerebrospinal venous insufficiency. J Cereb Blood Flow Metab 2013;33:657-668. 


\section{Neurology}

\section{Safety and efficacy of venoplasty in MS: A randomized, double-blind, sham-controlled phase II trial \\ Anthony L. Traboulsee, Lindsay Machan, J. Marc Girard, et al.}

Neurology 2018;91;e1660-e1668 Published Online before print September 28, 2018

DOI 10.1212/WNL.0000000000006423

This information is current as of September 28, 2018

\section{Updated Information \& Services}

References

Citations

Subspecialty Collections

Permissions \& Licensing

Reprints including high resolution figures, can be found at: http://n.neurology.org/content/91/18/e1660.full

This article cites 32 articles, 3 of which you can access for free at: http://n.neurology.org/content/91/18/e1660.full\#ref-list-1

This article has been cited by 5 HighWire-hosted articles: http://n.neurology.org/content/91/18/e1660.full\#\#otherarticles

This article, along with others on similar topics, appears in the following collection(s):

Class I

http://n.neurology.org/cgi/collection/class_1

Clinical trials Randomized controlled (CONSORT agreement) http://n.neurology.org/cgi/collection/clinical_trials_randomized_contro lled_consort_agreement

Multiple sclerosis

http://n.neurology.org/cgi/collection/multiple_sclerosis

Patient safety

http://n.neurology.org/cgi/collection/patient_safety

Information about reproducing this article in parts (figures,tables) or in its entirety can be found online at:

http://www.neurology.org/about/about_the_journal\#permissions

Information about ordering reprints can be found online:

http://n.neurology.org/subscribers/advertise

Neurology ${ }^{\circledR}$ is the official journal of the American Academy of Neurology. Published continuously since 1951, it is now a weekly with 48 issues per year. Copyright Copyright ( 2018 The Author(s). Published by Wolters Kluwer Health, Inc. on behalf of the American Academy of Neurology.. All rights reserved. Print ISSN: 0028-3878. Online ISSN: 1526-632X.

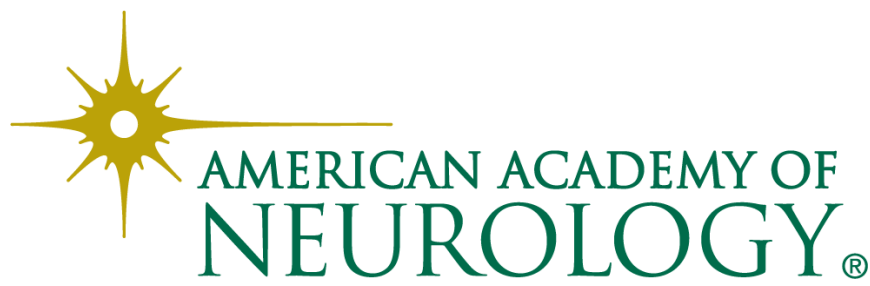

